\title{
A case report on the management of fetal supraventricular tachycardia
}

\author{
Meghana Vinod ${ }^{1}$, C. M. Neethu², S. Sudha ${ }^{3}$ \\ ${ }^{1}$ Amrita School of Pharmacy, Amrita Vishwa Vidyapeetham, AIMS Health Sciences Campus, Kochi, India. \\ ${ }^{2}$ Department of Pharmacy Practice, Amrita School of Pharmacy, Amrita Vishwa Vidyapeetham, AIMS Health Sciences Campus, Kochi, India. \\ ${ }^{3}$ Department of Obstetrics and Gynaecology, Amrita Institute of Medical Sciences and Research Centre, AIMS Health Sciences Campus, Kochi, India.
}

\begin{tabular}{|c|c|}
\hline ARTICLE INFO & ABSTRACT \\
\hline $\begin{array}{l}\text { Received on: } 04 / 06 / 2020 \\
\text { Accepted on: } 20 / 08 / 2020 \\
\text { Available online: } 05 / 12 / 2020\end{array}$ & $\begin{array}{l}\text { Fetal supraventricular tachycardia (SVT) has been found to have a prevalence ranging from } 1 / 1,000 \text { to } 1 / 25,000 \text { out } \\
\text { of all the pregnancies. However, if left untreated, it could cause significant cardiac failure of the fetus, leading to } \\
\text { fetal demise. Therefore, timely control of the fetal rhythm and its conversion to a normal sinus rhythm during the } \\
\text { gestation period itself is highly essential. Antiarrhythmic agents via the transplacental route have been described as }\end{array}$ \\
\hline $\begin{array}{l}\text { Key words: } \\
\text { Supraventricular } \\
\text { tachycardia, transplacental, } \\
\text { antiarrhythmic, fetal rhythm, } \\
\text { digoxin, flecainide. }\end{array}$ & $\begin{array}{l}\text { the mainstay for its rapid management. Hence, we present a case of transplacental antiarrhythmic treatment given for } \\
\text { the management of fetal SVT detected at } 22 \text { weeks of pregnancy. The diagnosis of fetal arrhythmia in a } 30 \text {-year-old } \\
\text { pregnant woman was established following which IV Digoxin along with a second line agent flecainide was chosen } \\
\text { and initiated immediately. Digoxin levels were maintained within the normal range of } 1.3-1.5 \mathrm{ng} / \mathrm{ml} \text { by appropriate } \\
\text { dosage adjustment. This multidrug approach was found to be effective in restoring the fetal rhythm and the patient was } \\
\text { discharged stably. The mother gave birth to a healthy female baby at } 36 \text { weeks of gestation. }\end{array}$ \\
\hline
\end{tabular}

\section{INTRODUCTION}

Fetal arrhythmias are found in at least $2 \%$ of all pregnancies during routine gynecological tests. Majority of them are usually benign, but certain fetal arrhythmias, such as supraventricular or ventricular tachycardia, atrial fibrillation, and atrioventricular (AV) block, may result in a low cardiac output, hydrops and death of the fetus. Fetal supraventricular tachycardia (SVT) is the most common type of fetal tachycardia (Husain et al., 2013). If left untreated, sustained FT could lead to an increase in central venous pressure and a decrease in cardiac output. This results in a fetal hemodynamic compromise which could further cause nonimmune hydrops fetalis leading to fetal demise.

SVT and atrial flutter (AF) are two of the most common types of sustained fetal arrhythmias. Control of the fetal rhythm

"Corresponding Author

C. M. Neethu, Department of Pharmacy Practice, Amrita School of Pharmacy, Amrita Vishwa Vidyapeetham, AIMS Health Sciences Campus, Kochi, India.E-mail: Neethucm92@gmail.com and its conversion to a normal sinus rhythm via transplacental medications was reported nearly 40 years ago (Çetin et al., 2014). Digoxin, flecainide, sotalol, amiodarone, and other antiarrhythmic agents have been found to have appreciable success rates in the treatment of fetal SVT in multiple studies. Here, we present a case of fetal SVT detected at 22 weeks of pregnancy in a 30-yearold woman. Transplacental therapy with digoxin and flecainide significantly restored the rhythm initially and prevented fetal mortality.

\section{Case report}

A 30-year-old healthy primi with a history of total thyroidectomy 3 years ago, for papillary carcinoma of thyroid, was admitted for regular FH monitoring at 22 weeks and 5 days of gestation. At initial evaluation, the fetus had atrial ectopics with short runs of SVT. Fetal heart rate was $233 \mathrm{bpm}$ with 1:1 atrioventricular (AV) conduction and long ventriculoatrial (VA) time. Follow-up after 1 week showed a persistent fetal SVT (long VA tachyarrhythmia suggestive of ectopic atrial tachycardia) with no hydrops and normal cardiac function. On investigating the mother's end organ functions, electrolytes and baseline electrocardiogram (ECG) were found to be normal. An opinion 
was sought from the Department of Pediatric Cardiology regarding the same. Periodic fetal echocardiography (echo) and ECG of the patient were taken and a diagnosis of fetal SVT was established. The patient was advised on transplacental therapy with flecainide and digoxin. Intravenous transplacental therapy was initiated with Digoxin 1,250 mcg for 24 hours along with oral flecainide $100 \mathrm{mg}$ thrice a day. After 24 hours, IV digoxin was converted to oral form at a dose of $0.25 \mathrm{mg}$ PO thrice a day. Endocrinology consultation was also obtained and the thyroxine dose was optimized to maintain the TSH level around $2 \mathrm{uIU} / \mathrm{ml}$. A significant fetal thyroid gland enlargement was ruled out in the fetal scan. Subsequent fetal echo done after 72 hours showed a sinus rhythm with just intermittent tachycardia. Maternal serum digoxin level was monitored, which was initially high $(2.75 \mathrm{ng} / \mathrm{ml})$ and the digoxin dose was reduced. Levels were repeated after 4 days and were found to be normal $(0.695 \mathrm{ng} / \mathrm{ml})$. Hence, oral digoxin was maintained at $0.25 \mathrm{mg}$ once daily. Serial fetal echo done also showed a sinus rhythm with intermittent tachycardia. Hence, oral digoxin and flecainide doses were optimized and maintained as follows: T. Digoxin 0.25 $\mathrm{mg}$ PO OD and T. Flecainide PO 50-50-50 mg. At discharge, the fetus was in sinus rhythm, maternal QTc interval normal, and the patient was hemodynamically stable. Later, the pregnancy was taken to term and a healthy, $2.58 \mathrm{~kg}$, female baby was delivered at 36 weeks and 1 day of gestation. The baby was well, in sinus rhythm post-delivery, by the mother's side and found to be feeding well. Both mother and baby were discharged without any further interventions.

\section{DISCUSSION}

Fetal SVT is defined as a 1:1 AV activity of the fetal heart rate (FHR) exceeding $200 \mathrm{bpm}$, accounting for up to $60 \%-80 \%$ of the total fetal tachyarrhythmias reported. It has a prevalence ranging from $1 / 1,000$ to $1 / 25,000$ of all pregnancies (Husain et al., 2013). Most of the fetal arrhythmias are usually of less clinical significance and result in favorable perinatal outcomes. However, supraventricular tachyarrhythmia can result in cardiac failure of the fetus along with fetal hydrops, Ballantyne's syndrome, and fetal demise (Ghenbot et al., 2019). Since most forms of fetal assessment and treatment are indirect, its management remains complicated. Ultrasound and echocardiography remain as the mainstay for evaluation and diagnosis, since external fetal monitoring alone cannot detect the high heart rates related to fetal tachycardia.

Transplacental therapy is the mainstay for management, unless there is some unusual contraindication to medicating the mother. The American Heart Association has published a statement recommending the generally acceptable agents and their dosing protocols (Donofrio et al., 2014). Digoxin (pregnancy category C) is the first-line therapy in non-hydropic fetuses, although it has limited utility in hydropic fetuses. It acts by increasing the refractoriness of the $\mathrm{AV}$ node and its therapeutic effect is due to its negative chronotropic and positive inotropic effects (Avari and Rhee, 2006). It has a long history of being the drug of first choice. Its safety, rapid oral absorption, ease of monitoring serum levels, and a reasonable response makes it effective in the treatment of fetal SVT. However, digoxin is not used alone in the treatment of fetal SVT due to its debatable effect in a reasonable time frame. It is usually used together with drugs like flecainide or sotalol.
Which second-line drug is to be selected depends on individual maternal and fetal factors such as gestational age, mechanism of arrhythmias, FHR and risk of prematurity, and degree of compromise (usually the presence of hydrops) (Jaeggi et al., 2011).

In our case, flecainide was used as the second-line agent. Both digoxin and flecainide were useful in the conversion of SVT to a normal rhythm as seen in most cases (Cheong, 2015; Porat et al., 2003). Flecanide (pregnancy category C) acts on accessory pathways, blocking conduction through sodium channels. It is effective in the hydropic population. Some institutions utilize flecanide as first-line therapy, with/without digoxin, for this group. The excellent fetal bioavailability, even in the presence of hydrops, makes flecanide attractive for transplacental therapy. However, it should be avoided in fetal AF and mothers with structural or ischemic heart disease, cardiomyopathy, or bradycardia. Major side effects include maternal proarrhythmia and QRS prolongation. There are reports of fetal demise in patients treated with flecanide, but it is unclear whether the cause of death was proarrhythmia or the severity of the heart failure (Avari and Rhee, 2006). Contemporary data, unlike in our case, also suggest good effects with sotalol or amiodarone. Amiodarone is typically used in refractory cases since it poses a high risk of toxicity, including hypothyroidism and fetal goitre (Malhamé et al., 2019). On the other hand, sotalol is associated with a higher rate of termination of fetal atrial fibrillation (AF), with some modest effects on tachycardia. This implies that in future, combining sotalol with either digoxin or flecainide as first-line therapy could give a better chance of terminating fetal tachyarrhythmias. The safety and efficacy of a combined drug treatment need to be evaluated in a larger prospective study. In our case, the maternal maintenance dose was determined by titrating to the fetal response. To avoid any maternal toxicity such as gastrointestinal and central nervous system disturbances, arrhythmias including premature beats and AV block, close inpatient maternal monitoring was necessary. Daily ECGs were done in order to monitor for PR prolongation and T-wave changes. Another factor in the following of digoxin therapy is the blood level of the drug. A blood level exceeding the range of $1.3-1.5 \mathrm{ng} / \mathrm{ml}$ could indicate signs of digoxin toxicity. In our case, the serum digoxin level was found to be within the normal range $(0.695 \mathrm{ng} / \mathrm{ml})$ at $0.25 \mathrm{mg}$ once daily dose of digoxin, with no signs of toxicity present during further follow-up.

A similar case of fetal SVT was reported in a 31-yearold healthy primigravid woman at 13 weeks of gestation. As in our case, digoxin and flecainide transplacental therapy was found to have a fast and effective response in its management (Porat et al., 2003). Another case was reported in an 18-year-old woman at 25 weeks of gestation. Here, unlike our case, sotalol therapy along with digoxin was found to be successful in the resolution of hydrops and conversion into a normal fetal sinus rhythm (Merriman et al., 2008). Similar to our case, a normal delivery can be planned if the fetus is maintained on sinus rhythm, which is the most likely outcome in case of fetal SVTs. However, if sustained tachycardia is existent, either a cardiac or pediatric specialist should be available at the time of delivery to help with diagnosis and therapeutic decision-making. Moreover, if SVT is not controlled, then intrauterine delivery is warranted, especially from 28 weeks of pregnancy after complete lung maturity. 
Our case report indicates the significance of a multidisciplinary treatment approach using transplacental therapy in the appropriate and timely management of tachyarrhythmia at the fetal stage. Nevertheless, it is necessary for researchers to conduct further studies and formulate a suitable treatment protocol in the management of fetal tachyarrhythmia.

\section{CONCLUSION}

The multidrug approach with antiarrhythmic agents is the mainstay in the suitable treatment of fetal SVT and can be managed successfully without any cardiac complications.

\section{INFORMED CONSENT}

Informed consent was duly signed and obtained from the patient.

\section{CONFLICT OF INTEREST}

The authors have no conflict of interest in connection with this paper. This case report did not receive any funding from any organization.

\section{FUNDING}

None.

\section{REFERENCES}

Avari JN, Rhee EK. Diagnosis and treatment of fetal tachyarrhythmias. US Cardiol Dis, 2006; 3:1.

Çetin C, Akçabay Ç, Büyükkurt S, Özbarlas N. Successful medical treatment of fetal supraventricular tachycardia that cause hydrops fetalis. Turk J Obstet Gynecol, 2014; 11:193.

Cheong MU. Intrauterine management of fetal supraventricular tachycardia (SVT) with cardiac failure. Med J Malaysia, 2015; 706:371.

Donofrio MT, Moon-Grady AJ, Hornberger LK, Copel JA, Sklansky MS, Abuhamad A, Cuneo BF, Huhta JC, Jonas RA, Krishnan A,
Lacey S, Lee W, Michelfelder EC, Sr Rempel GR, Silverman NH, Spray TL, Strasburger JF, Tworetzky W, Rychik J, American Heart Association Adults With Congenital Heart Disease, Joint Committee of the Council on Cardiovascular Disease in the Young, and Council on Clinical Cardiology, Council on Cardiovascular Surgery and Anesthesia, and Council on Cardiovascular and Stroke Nursing. Diagnosis and treatment of fetal cardiac disease: a scientific statement from the American Heart Association. Circulation, 2014; 129:2183-242.

Ghenbot R, Gonzalez-Brown V, Markham K. Management of fetal supraventricular tachycardia: three cases at a single institution. Obstet Gynecol Cases Rev, 2019; 6:146;

Husain A, Hubail Z, Al Banna R. Fetal supraventricular tachycardia, treating the baby by targeting the mother. BMJ Case Rep, 2013; 2013:bcr2012008515;

Jaeggi ET, Carvalho JS, De Groot E, Api O, Clur SA, Rammeloo L, McCrindle BW, Ryan G, Manlhiot C, Blom NA. Comparison of transplacental treatment of fetal supraventricular tachyarrhythmias with digoxin, flecainide, and sotalol: results of a nonrandomized multicenter study. Circulation, 2011; 124:1747-54.

Malhamé I, Gandhi C, Tarabulsi G, Esposito M, Lombardi K, Chu A, Chen KK. Maternal monitoring and safety considerations during antiarrhythmic treatment for fetal supraventricular tachycardia. Obstet Med, 2019; $12: 66-75$.

Merriman JB, Gonzalez JM, Rychik J, Ural SH. Can digoxin and sotalol therapy for fetal supraventricular tachycardia and hydrops be successful? A case report. J Reprod Med, 2008; 53:357-9.

Porat S, Anteby EY, Hamani Y, Yagel S. Fetal supraventricular tachycardia diagnosed and treatedat 13 weeks of gestation: a case report. Ultrasound Obstet Gynecol, 2003; 21:302-5.

How to cite this article:

Vinod M, Neethu CM, Sudha S. A case report on the management of fetal supraventricular tachycardia. J Appl Pharm Sci, 2020; 10(12):111-113. 